\title{
THE RESEARCH JOURNAL TRANSPORT: PEER-REVIEWING PROCESS IN 2017
}

\author{
Olegas Prentkovskis ${ }^{1}$, Raimundas Junevičius ${ }^{2}$, Paulius Skačkauskas ${ }^{3}$ \\ ${ }^{1}$ Editor-in-Chief of the Research Journal TRANSPORT, E-mail: olegas.prentkovskis@vgtu.lt \\ ${ }^{2}$ Deputy Editor-in-Chief of the Research Journal TRANSPORT, E-mail: raimundas.junevicius@vgtu.lt \\ ${ }^{3}$ Managing Editor of the Research Journal TRANSPORT, E-mail: paulius.skackauskas@vgtu.lt
}

3 November 2017

At present, transport is one of the key branches playing a crucial role in the development of economy. Reliable and properly organized transport services are required for a professional performance of industry, construction and agriculture. The public mood and efficiency of work also largely depend on the valuable functions of a carefully chosen transport system. A steady increase in transportation is accompanied by growing demands for a higher quality of transport services and optimum efficiency of transport performance. Currently, joint efforts taken by the transport experts and governing institutions of the country are required to develop and enhance the performance of the national transport system conducting theoretical and empirical research.

TRANSPORT is an international peer-reviewed journal covering main aspects of transport and providing a source of information for the engineer and the applied scientist. The journal TRANSPORT publishes articles in the fields of transport policy; fundamentals of the transport system; technology for carrying passengers and freight using road, railway, inland waterways, sea and air transport; technology for multimodal transportation and logistics; loading technology; roads, railways; airports, ports; traffic safety and environment protection; design, manufacture and exploitation of motor vehicles; transport energetics; fuels, lubricants and maintenance materials; teamwork of customs and transport; transport information technologies; transport economics and management; transport standards; transport educology and history, etc.

To peer-review manuscript submissions, the research journal TRANSPORT uses the interactive system ScholarOne Manuscripts (formerly known as Manuscript Central, http://mc.manuscriptcentral.com/stra).

All manuscripts submitted through ScholarOne Manuscripts are peer-reviewed by members of the Editorial Board or appointed experts. The minimum number of score sheets (peer-review forms filled by reviewers for each submitted manuscript) amounts to three and the average number of score sheets (peer-review forms) is 3.4 (in 2017).

In 2011-2017 the research journal TRANSPORT has been published by Vilnius Gediminas Technical University in partnership with the Lithuanian Academy of Science and Taylor \& Francis. Decision ratios of the submitted manuscripts in 2011-2017 (source: TRANSPORT ScholarOne Manuscripts, data until 3 November 2017) are presented on Fig. 1. Acceptance ratios of the submitted manuscripts in 2011-2017 (source: TRANSPORT ScholarOne Manuscripts, data until 3 November 2017) are presented on Fig. 2. Decision ratios of the submitted manuscripts in 2017 (source: TRANSPORT ScholarOne Manuscripts, data until 3 November 2017) are presented on Fig. 3.

Success and quality of the research journal TRANSPORT depends to a large extent on the activity and performance of its Editorial Board and Reviewers. Reviewers are scientists who volunteer to contribute to the improvement of the quality of transport engineering and transport management fields by guiding authors in their efforts to publish their research outputs that help to enlarge the scientific content of the research journal TRANSPORT, for which we feel truly grateful.

Thus, on behalf of the Editorial Board of the research journal TRANSPORT we would like to express our gratitude to the following reviewers (Table) for their important contribution to the quality of scientific articles submitted in 2014-2017 (accepted in 2015-2017), which were peer-reviewed and part of them - published in TRANSPORT 32(1, 2, 3, 4) 2017 or as 'Article in Press' (Latest Articles on www).

Corresponding author: Olegas Prentkovskis

E-mail: olegas.prentkovskis@vgtu.lt

Copyright $\odot 2017$ Vilnius Gediminas Technical University (VGTU) Press

http://www.tandfonline.com/TRAN 

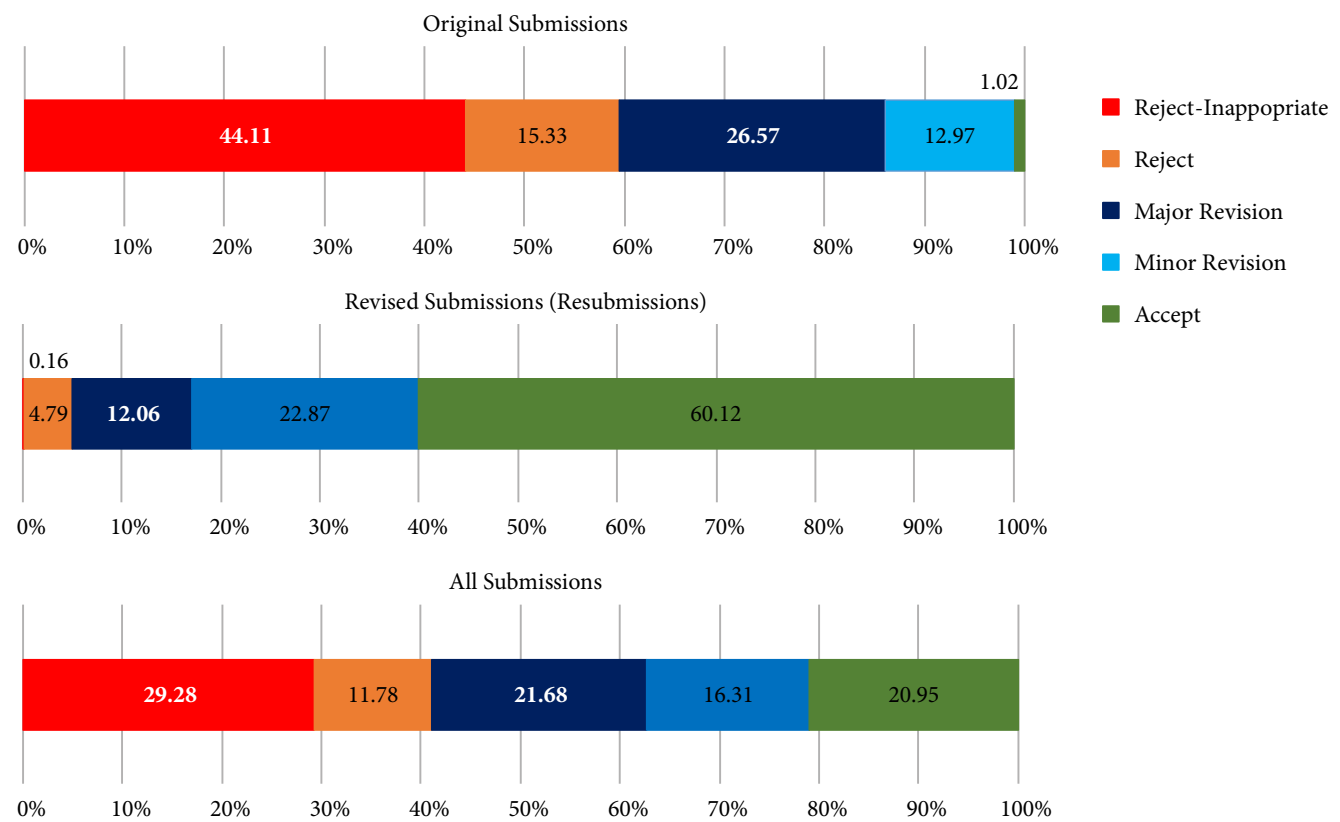

Fig. 1. Decision ratios of the submitted manuscripts in 2011-2017

(note: 'revised submissions' follows 'minor revision' or 'major revision' after 'original submissions')

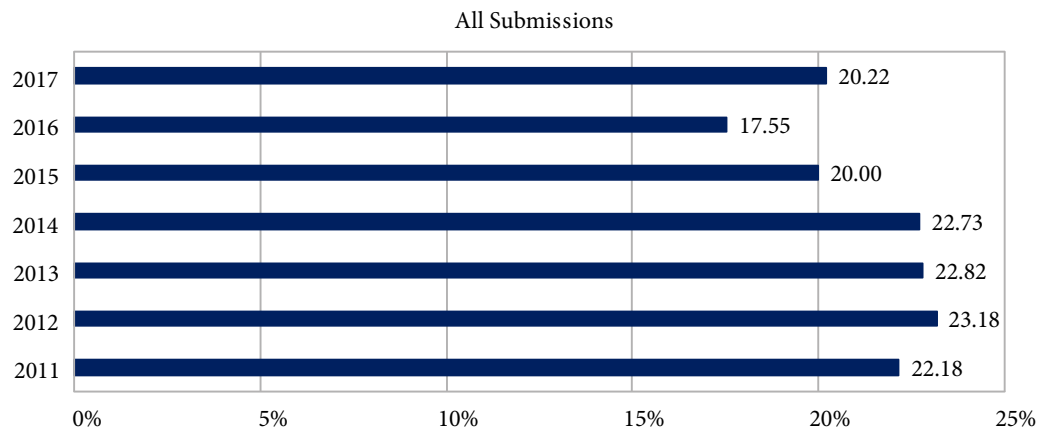

Fig. 2. Acceptence ratios of the submitted manuscripts in 2011-2017
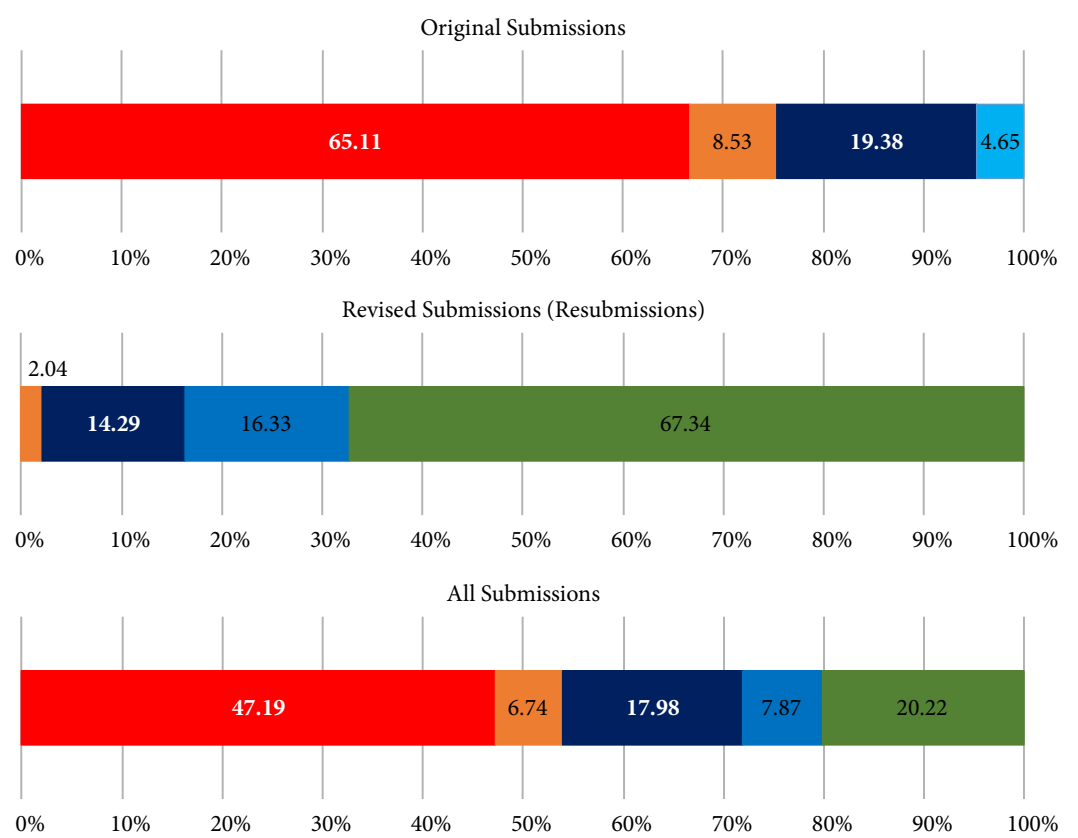

Fig. 3. Decision ratios of the submitted manuscripts in 2017

(note: 'revised submissions' follows 'minor revision' or 'major revision' after 'original submissions') 
Table. List of the reviewers of the scientific peer-reviewed articles submitted in 2014-2017 (accepted in 2015-2017 and published in TRANSPORT 32(1, 2, 3, 4) 2017 or as 'Article in Press')

\begin{tabular}{|c|c|}
\hline Surname, Name & Institution \\
\hline \multicolumn{2}{|r|}{ Australia } \\
\hline Spiryagin, Maksym & \multirow[t]{2}{*}{ Central Queensland University } \\
\hline Sun, Yan & \\
\hline Burdett, Robert & \multirow[t]{3}{*}{ Queensland University of Technology } \\
\hline Casey, Bradley & \\
\hline Yarlagadda, Prasad & \\
\hline Hutchinson, Paul & University of Adelaide \\
\hline Bambach, Mike & University of New South Wales \\
\hline Taylor, Michael & University of South Australia \\
\hline Österle, Ines & University of Sydney Business School \\
\hline Assemi, Behrang & \multirow[t]{3}{*}{ University of Queensland } \\
\hline Prato, Carlo & \\
\hline Tavassoli, Ahmad & \\
\hline \multicolumn{2}{|r|}{ Austria } \\
\hline Lindorfer, Manuel & $\begin{array}{l}\text { University of Applied Sinces Upper } \\
\text { Austria }\end{array}$ \\
\hline Edelmann, Johannes & \multirow[t]{2}{*}{ Vienna University of Technology } \\
\hline Schulz, Alexander & \\
\hline \multicolumn{2}{|r|}{ Belgium } \\
\hline Janjevic, Milena & Free University of Brussels \\
\hline Verlinde, Sara & Ghent University \\
\hline Cools, Mario & University of Liège \\
\hline Kouroussis, Georges & University of Mons \\
\hline \multicolumn{2}{|r|}{ Brazil } \\
\hline Carlson, Rodrigo & Federal University Santa Catarina \\
\hline Morooka, Celso & University of Campinas \\
\hline \multicolumn{2}{|r|}{ Bulgaria } \\
\hline Ivanov, Rosen & 'Angel Kanchev' University of Ruse \\
\hline \multicolumn{2}{|r|}{ Canada } \\
\hline Alecsandru, Ciprian & Concordia University \\
\hline El-Geneidy, Ahmed & McGill University \\
\hline Huang, Kai & McMaster University \\
\hline Trépanier, Martin & Polytechnic of Montréal \\
\hline Yin, Derek & University of Alberta \\
\hline Kabir, Golam & University of British Columbia \\
\hline Kattan, Lina & University of Calgary \\
\hline $\mathrm{Ng}$, Adolf & University of Manitoba \\
\hline Garmsiri, Shahryar & $\begin{array}{l}\text { University of Ontario Institute } \\
\text { of Technology }\end{array}$ \\
\hline $\begin{array}{l}\text { Younes, Maram } \\
\text { Bani }\end{array}$ & University of Ottawa \\
\hline Dai, Li Ming & University of Regina \\
\hline Bachmann, Chris & University of Toronto \\
\hline Lee, Chris & University of Windsor \\
\hline
\end{tabular}

\begin{tabular}{|c|c|}
\hline Surname, Name & Institution \\
\hline \multicolumn{2}{|r|}{ Colombia } \\
\hline $\begin{array}{l}\text { Arango Serna, } \\
\text { Martin Dario }\end{array}$ & National University of Colombia \\
\hline \multicolumn{2}{|r|}{ Chile } \\
\hline Larrain, Homero & Pontifical Catholic University of Chile \\
\hline \multicolumn{2}{|r|}{ China } \\
\hline Li, Qi-Lang & Anhui Jianzhu University \\
\hline Ma, Weijia & $\begin{array}{l}\text { AVIC Aerodynamics Research } \\
\text { Institute }\end{array}$ \\
\hline Li, Hongqi & Beihang University \\
\hline Bin, Xin & Beijing Institute of Technology \\
\hline Chen, Shaokuan & \multirow[t]{5}{*}{ Beijing Jiaotong University } \\
\hline Gao, Liang & \\
\hline Guan, Wei & \\
\hline Liu, Jiang & \\
\hline Shi, Jin & \\
\hline Zhao, Xiaohua & Beijing University of Technology \\
\hline Peng, Yong & Central South University \\
\hline Wang, Yonggang & Chang'an University \\
\hline Zhang, Zhichao & China Academy of Railway Sciences \\
\hline Bian, Zhan & \multirow[t]{3}{*}{ Dalian Maritime University } \\
\hline Yu, Bin & \\
\hline Zeng, Qingcheng & \\
\hline Lu, Jing & \multirow[t]{3}{*}{ Dalian University of Technology } \\
\hline $\mathrm{Xu}$, Hongfeng & \\
\hline Zhang, Lihui & \\
\hline Gu, Guangxin & Fudan University \\
\hline Bie, Yiming & Harbin Institute of Technology \\
\hline Hou, Shujuan & Hunan University \\
\hline Wang, Tianxing & $\begin{array}{l}\text { Institute of Remote Sensing and } \\
\text { Digital Earth }\end{array}$ \\
\hline Yang, Xinfeng & Lanzhou Jiaotong University \\
\hline Lu, Ai-Zhong & $\begin{array}{l}\text { North China Electric Power } \\
\text { University }\end{array}$ \\
\hline Cai, Zhiqiang & \multirow{2}{*}{$\begin{array}{l}\text { Northwestern Polytechnical } \\
\text { University }\end{array}$} \\
\hline Chen, Jian-Zhong & \\
\hline Liu, Yu & Peking University \\
\hline An, Xiaomi & Renmin University of China \\
\hline Lu, Chunxia & Shanghai Jiaotong University \\
\hline Ge, Ying-En & Shanghai Maritime University \\
\hline Wei, Gui-wu & Sichuan Normal University \\
\hline Cheng, Jian-Chuan & \multirow[t]{3}{*}{ Southeast University } \\
\hline Li, Zhibin & \\
\hline Wang, Weize & \\
\hline
\end{tabular}




\begin{tabular}{|c|c|}
\hline Surname, Name & Institution \\
\hline Jin, Xuesong & \multirow[t]{3}{*}{ Southwest Jiaotong University } \\
\hline Yao, Yuan & \\
\hline Zhai, Wanming & \\
\hline Liu, Run & Tianjin University \\
\hline Lun, Zhang & \multirow[t]{2}{*}{ Tongji University } \\
\hline Zhang, Xiaoning & \\
\hline Wang, Yueshe & Xi'an Jiaotong University \\
\hline Wang, Fujian & Zhejiang University \\
\hline \multicolumn{2}{|r|}{ Croatia } \\
\hline Pupavac, Drago & Polytechnic of Rijeka \\
\hline Krile, Srecko & University of Dubrovnik \\
\hline $\begin{array}{l}\text { Deluka-Tibljaš, } \\
\text { Aleksandra }\end{array}$ & \multirow[t]{3}{*}{ University of Rijeka } \\
\hline Hess, Mirano & \\
\hline Tijan, Edvard & \\
\hline Breski, Deana & University of Split \\
\hline Abramović, Borna & \multirow[t]{7}{*}{ University of Zagreb } \\
\hline Anžek, Mario & \\
\hline Barić, Danijela & \\
\hline Ćavar, Ivana & \\
\hline Lakušić, Stjepan & \\
\hline Peraković, Dragan & \\
\hline Sostaric, Marko & \\
\hline \multicolumn{2}{|r|}{ Czech Republic } \\
\hline Daněk, Vladimír & \multirow[t]{4}{*}{ Brno University of Technology } \\
\hline Porteš, Petr & \\
\hline Štigler, Jaroslav & \\
\hline Vosecký, Slavomír & \\
\hline Čarská, Zuzana & \multirow[t]{2}{*}{ Czech Technical University in Prague } \\
\hline Janoš, Vít & \\
\hline Jakubicek, Paul & Freight Transport Research Institute \\
\hline Brabec, Marek & $\begin{array}{l}\text { Institute of Computer Science of the } \\
\text { Czech Academy of Sciences }\end{array}$ \\
\hline Stopka, Ondrej & $\begin{array}{l}\text { Institute of Technology and Business } \\
\text { in České Budějovice }\end{array}$ \\
\hline Soukopová, Jana & Masaryk University \\
\hline Kolar, Vaclav & \multirow[t]{2}{*}{ Technical University of Ostrava } \\
\hline Stýskala, Vítězslav & \\
\hline Drahotský, Ivo & \multirow[t]{5}{*}{ University of Pardubice } \\
\hline Lata, Michael & \\
\hline Lopour, Pavel & \\
\hline Soušek, Radovan & \\
\hline Švadlenka, Libor & \\
\hline Kraft, Stanislav & $\begin{array}{l}\text { University of South Bohemia } \\
\text { in České Budějovice }\end{array}$ \\
\hline
\end{tabular}

\begin{tabular}{|c|c|}
\hline Surname, Name & Institution \\
\hline \multicolumn{2}{|r|}{ Denmark } \\
\hline Anderson, Marie & \multirow[t]{4}{*}{ Technical University of Denmark } \\
\hline $\begin{array}{l}\text { Gudmundsson, } \\
\text { Henrik }\end{array}$ & \\
\hline Mulalic, Ismir & \\
\hline Rasmussen, Thomas & \\
\hline \multicolumn{2}{|r|}{ Estonia } \\
\hline $\begin{array}{l}\text { Segercrantz, } \\
\text { Wladimir }\end{array}$ & $\begin{array}{l}\text { Tallinn University of Applied } \\
\text { Sciences }\end{array}$ \\
\hline Aavik, Andrus & \multirow[t]{2}{*}{ Tallinn University of Technology } \\
\hline Antov, Dago & \\
\hline \multicolumn{2}{|r|}{ Ethiopia } \\
\hline Beshah, Tibebe & Addis Ababa University \\
\hline \multicolumn{2}{|r|}{ Finland } \\
\hline Bruun, Eric & \multirow[t]{3}{*}{ Aalto University } \\
\hline Matusiak, Jerzy & \\
\hline Siikonen, Timo & \\
\hline Salminen, Jani & Finnish Environment Institute \\
\hline Liimatainen, Heikki & Tampere University of Technology \\
\hline Tapaninen, Ulla & University of Turku \\
\hline \multicolumn{2}{|r|}{ France } \\
\hline Herrero, German & Atos S.A. \\
\hline Khoudour, Louahdi & CEREMA \\
\hline Bhouri, Neïla & $\begin{array}{l}\text { French Institute of Science and } \\
\text { Technology for Transport, Spatial } \\
\text { Planning, Development and } \\
\text { Networks }\end{array}$ \\
\hline $\begin{array}{l}\text { Gonzalez-Feliu, } \\
\text { Jesus }\end{array}$ & Mines School of Saint-Étienne \\
\hline \multicolumn{2}{|r|}{ Germany } \\
\hline Heinitz, Florian & Erfurt University of Applied Science \\
\hline Ivanov, Valentin & Ilmenau University of Technology \\
\hline Krüger, Philip & Technical University of Darmstadt \\
\hline $\begin{array}{l}\text { Antoniou, } \\
\text { Constantinos }\end{array}$ & Technical University of Munich \\
\hline \multicolumn{2}{|r|}{ Greece } \\
\hline Samaras, Zisis & Aristotle University of Thessaloniki \\
\hline Bekiaris, Evangelos & \multirow{7}{*}{$\begin{array}{l}\text { Center for Research and Technology } \\
\text { Hellas }\end{array}$} \\
\hline $\begin{array}{l}\text { Chaniotakis, } \\
\text { Emmanouil }\end{array}$ & \\
\hline $\begin{array}{l}\text { Giannopoulos, } \\
\text { George Anastasios }\end{array}$ & \\
\hline Mintsis, Vagelis & \\
\hline $\begin{array}{l}\text { Salanova Grau, } \\
\text { Josep Maria }\end{array}$ & \\
\hline Stamos, Iraklis & \\
\hline $\begin{array}{l}\text { Stathacopoulos, } \\
\text { Alexander }\end{array}$ & \\
\hline
\end{tabular}




\begin{tabular}{|c|c|}
\hline Surname, Name & Institution \\
\hline $\begin{array}{l}\text { Georgoudas, } \\
\text { Ioakeim }\end{array}$ & Democritus University of Thrace \\
\hline Loizos, Andreas & \multirow{3}{*}{$\begin{array}{l}\text { National Technical University } \\
\text { of Athens }\end{array}$} \\
\hline $\begin{array}{l}\text { Theofilatos, } \\
\text { Athanasios }\end{array}$ & \\
\hline Yannis, George & \\
\hline $\begin{array}{l}\text { Psomopoulos, } \\
\text { Constantinos }\end{array}$ & $\begin{array}{l}\text { Technological Education Institute } \\
\text { of Piraeus }\end{array}$ \\
\hline Tzannatos, Ernestos & University of Piraeus \\
\hline Nikitakos, Nikitas & University of the Aegean \\
\hline $\begin{array}{l}\text { Vogiatzis, } \\
\text { Konstantinos }\end{array}$ & University of Thessaly \\
\hline \multicolumn{2}{|r|}{ Hong Kong } \\
\hline $\begin{array}{l}\text { Yuen, Kevin Kam } \\
\text { Fung }\end{array}$ & \multirow[t]{2}{*}{ Hong Kong Polytechnic University } \\
\hline Zhu, Wenting & \\
\hline Huang, George & \multirow[t]{2}{*}{ University of Hong Kong } \\
\hline Szeto, Wai Yuen & \\
\hline \multicolumn{2}{|r|}{ Hungary } \\
\hline Bereczky, Ákos & \multirow{7}{*}{$\begin{array}{l}\text { Budapest University of Technology } \\
\text { and Economics }\end{array}$} \\
\hline Bokor, Zoltan & \\
\hline Csiszár, Csaba & \\
\hline Tanczos, Katalin & \\
\hline Tettamanti, Tamás & \\
\hline Török, Ádám & \\
\hline Török, Árpád & \\
\hline Gaspar, Peter & $\begin{array}{l}\text { Computer and Automation Research } \\
\text { Institute }\end{array}$ \\
\hline Zöldy, Máté & MOL DS Product Development \\
\hline \multicolumn{2}{|r|}{ India } \\
\hline $\begin{array}{l}\text { Adil, Gajendra } \\
\text { Kumar }\end{array}$ & \multirow[t]{3}{*}{$\begin{array}{l}\text { Indian Institute of Technology } \\
\text { Bombay }\end{array}$} \\
\hline Sangole, Jayant & \\
\hline Singh, Dharamveer & \\
\hline Pandit, Debapratim & $\begin{array}{l}\text { Indian Institute of Technology } \\
\text { Kharagpur }\end{array}$ \\
\hline Vanajakshi, Lelitha & $\begin{array}{l}\text { Indian Institute of Technology } \\
\text { Madras }\end{array}$ \\
\hline Bhuyan, Prasanta & $\begin{array}{l}\text { National Institute of Technology } \\
\text { Rourkela }\end{array}$ \\
\hline \multicolumn{2}{|r|}{ Iran } \\
\hline Esmaeili, Ahmad & Allameh Tabataba'i University \\
\hline Razaghi, Reza & $\begin{array}{l}\text { Iran University of Science and } \\
\text { Technology }\end{array}$ \\
\hline $\begin{array}{l}\text { Shafabakhsh, } \\
\text { Gholamali }\end{array}$ & Semnan University \\
\hline Abdi, Javad & University of Tehran \\
\hline Taghavifar, Hamid & Urmia University \\
\hline
\end{tabular}

\begin{tabular}{|c|c|}
\hline Surname, Name & Institution \\
\hline \multicolumn{2}{|r|}{ Ireland } \\
\hline Enright, Bernard & Dublin Institute of Technology \\
\hline Chen, I-Shuo & University of Dublin Trinity College \\
\hline \multicolumn{2}{|r|}{ Israel } \\
\hline Bekhor, Shlomo & \multirow{2}{*}{$\begin{array}{l}\text { Technion - Israel Institute of } \\
\text { Technology }\end{array}$} \\
\hline Gitelman, Victoria & \\
\hline \multicolumn{2}{|r|}{ Italy } \\
\hline Di Pasquale, Guide & Pluservice srl. \\
\hline Melacini, Marco & Polytechnic University of Milan \\
\hline Pronello, Cristina & Polytechnic University of Turin \\
\hline Cantisani, Giuseppe & \multirow[t]{2}{*}{ Sapienza University of Rome } \\
\hline $\begin{array}{l}\text { Loprencipe, } \\
\text { Giuseppe }\end{array}$ & \\
\hline Cipriani, Ernesto & Roma Tre University \\
\hline Bortolini, Marco & \multirow[t]{3}{*}{ University of Bologna } \\
\hline Simone, Andrea & \\
\hline Vignali, Valeria & \\
\hline Mazzulla, Gabriella & University of Calabria \\
\hline D’Apuzzo, Mauro & University of Cassino \\
\hline Meli, Enrico & \multirow[t]{2}{*}{ University of Florence } \\
\hline Pugi, Luca & \\
\hline Pellegrino, Orazio & \multirow[t]{2}{*}{ University of Messina } \\
\hline Sollazzo, Giuseppe & \\
\hline $\begin{array}{l}\text { Hadjidimitriou, } \\
\text { Selini }\end{array}$ & \multirow[t]{2}{*}{$\begin{array}{l}\text { University of Modena and Reggio } \\
\text { Emilia }\end{array}$} \\
\hline Novellani, Stefano & \\
\hline $\begin{array}{l}\text { Abbondati, } \\
\text { Francesco }\end{array}$ & \multirow[t]{6}{*}{ University of Naples Federico II } \\
\hline $\begin{array}{l}\text { Biancardo, Salvatore } \\
\text { Antonio }\end{array}$ & \\
\hline $\begin{array}{l}\text { Busiello, } \\
\text { Mariarosaria }\end{array}$ & \\
\hline De Luca, Mario & \\
\hline $\begin{array}{l}\text { Dell'Acqua, } \\
\text { Gianluca }\end{array}$ & \\
\hline Russo, Francesca & \\
\hline Battiato, Andrea & University of Padua \\
\hline Granà, Anna & University of Palermo \\
\hline Giuliani, Felice & University of Parma \\
\hline Nicolosi, Vittorio & University of Rome Tor Vergata \\
\hline Basile, Francesco & University of Salerno \\
\hline Mauro, Raffaele & University of Trento \\
\hline Danielis, Romeo & \multirow[t]{3}{*}{ University of Trieste } \\
\hline $\begin{array}{l}\text { Francescutto, } \\
\text { Alberto }\end{array}$ & \\
\hline Ukovich, Walter & \\
\hline
\end{tabular}




\begin{tabular}{|c|c|}
\hline Surname, Name & Institution \\
\hline \multicolumn{2}{|r|}{ Japan } \\
\hline Munehiro, Kazunori & $\begin{array}{l}\text { Civil Engimeering Research Institute } \\
\text { for Cold Region }\end{array}$ \\
\hline Uchida, Kenetsu & Hokkaido University \\
\hline Li, Guoquan & Railway Technical Research Institute \\
\hline Mishina, Tsutomu & Seibi University Junior College \\
\hline Shintani, Koichi & Tokai University \\
\hline Paul, Topon & Toshiba Corporation \\
\hline \multicolumn{2}{|r|}{ Jordan } \\
\hline Al-Omari, Bashar & $\begin{array}{l}\text { Jordan University of Science and } \\
\text { Technology }\end{array}$ \\
\hline \multicolumn{2}{|r|}{ Korea } \\
\hline Son, Joonwoo & $\begin{array}{l}\text { Daegu Gyeongbuk Institute of } \\
\text { Science and Technology }\end{array}$ \\
\hline Park, Simsoo & Korea University \\
\hline Kim, Seon Jin & Pukyong National University \\
\hline Ko, Joonho & Seoul Institute \\
\hline \multicolumn{2}{|r|}{ Latvia } \\
\hline Karevs, Vladimirs & \multirow[t]{3}{*}{ Riga Technical University } \\
\hline Smirnovs, Juris & \\
\hline Viba, Janis & \\
\hline Alomar, Iyad & \multirow{3}{*}{$\begin{array}{l}\text { Transport and Telecommunication } \\
\text { Institute }\end{array}$} \\
\hline Kabashkin, Igor & \\
\hline $\begin{array}{l}\text { Yatskiv (Jackiva), } \\
\text { Irina }\end{array}$ & \\
\hline \multicolumn{2}{|r|}{ Lithuania } \\
\hline Jotautiené, Eglè & \multirow[t]{3}{*}{ Aleksandras Stulginskis University } \\
\hline $\begin{array}{l}\text { Makarevičienè, } \\
\text { Violeta }\end{array}$ & \\
\hline Žunda, Audrius & \\
\hline Bazaras, Žilvinas & \multirow[t]{8}{*}{ Kaunas University of Technology } \\
\hline $\begin{array}{l}\text { Ilgakojytè- } \\
\text { Bazarienė, Jurga }\end{array}$ & \\
\hline $\begin{array}{l}\text { Kandrotaitè- } \\
\text { Janutienė, Rasa }\end{array}$ & \\
\hline Keršys, Artūras & \\
\hline Keršys, Robertas & \\
\hline Kliučinskas, Linas & \\
\hline Makaras, Rolandas & \\
\hline $\begin{array}{l}\text { Raslavičius, } \\
\text { Laurencas }\end{array}$ & \\
\hline Janutènienè, Jolanta & \multirow[t]{3}{*}{ Klaipèda University } \\
\hline Lebedevas, Sergejus & \\
\hline Smailys, Vytautas & \\
\hline Rudzkienè, Vitalija & Mykolas Romeris University \\
\hline
\end{tabular}

\begin{tabular}{|c|c|}
\hline Surname, Name & Institution \\
\hline $\begin{array}{l}\text { Antuchevičiené, } \\
\text { Jurgita }\end{array}$ & \multirow[t]{38}{*}{$\begin{array}{l}\text { Vilnius Gediminas } \\
\text { Technical University }\end{array}$} \\
\hline Bazaras, Darius & \\
\hline $\begin{array}{l}\text { Bogdevičius, } \\
\text { Marijonas }\end{array}$ & \\
\hline Braziūnas, Justas & \\
\hline Bučinskas, Vytautas & \\
\hline Bureika, Gintautas & \\
\hline $\begin{array}{l}\text { Burinskiené, } \\
\text { Aurelija } \\
\end{array}$ & \\
\hline Burinskienè, Marija & \\
\hline Čižiūnienė, Kristina & \\
\hline Dailydka, Stasys & \\
\hline Davidavičienė, Vida & \\
\hline Gribniak, Viktor & \\
\hline Grigonis, Vytautas & \\
\hline Griškevičius, Julius & \\
\hline Jasiūnienė, Vilma & \\
\hline $\begin{array}{l}\text { Kondroška, } \\
\text { Vaidotas }\end{array}$ & \\
\hline $\begin{array}{l}\text { Liudvinavičius, } \\
\text { Lionginas }\end{array}$ & \\
\hline Oginskas, Rolandas & \\
\hline Palšaitis, Ramūnas & \\
\hline Pečeliūnas, Robertas & \\
\hline Petrenko, Viačeslav & \\
\hline \begin{tabular}{|l} 
Podvezko, \\
Valentinas
\end{tabular} & \\
\hline $\begin{array}{l}\text { Pukalskas, } \\
\text { Saugirdas }\end{array}$ & \\
\hline Rimkus, Alfredas & \\
\hline Rudinskas, Darius & \\
\hline $\begin{array}{l}\text { Sivilevičius, } \\
\text { Henrikas }\end{array}$ & \\
\hline Skorupa, Pavel & \\
\hline Skrickij, Viktor & \\
\hline Sokolovskij, Edgar & \\
\hline Udris, Dainius & \\
\hline $\begin{array}{l}\text { Ušpalytė- } \\
\text { Vitkūnienė, Rasa }\end{array}$ & \\
\hline Vaidogas, Egidijus & \\
\hline Vaišis, Vaidotas & \\
\hline Vaitkus, Audrius & \\
\hline Valivonis, Juozas & \\
\hline $\begin{array}{l}\text { Zavadskas, } \\
\text { Edmundas }\end{array}$ & \\
\hline Žilionienė, Daiva & \\
\hline Žuraulis, Vidas & \\
\hline
\end{tabular}




\begin{tabular}{|c|c|}
\hline Surname, Name & Institution \\
\hline Štreimikienè, Dalia & Vilnius University \\
\hline \multicolumn{2}{|r|}{ Malaysia } \\
\hline $\begin{array}{l}\text { Gunawan, Teddy } \\
\text { Surya }\end{array}$ & $\begin{array}{l}\text { International Islamic University } \\
\text { Malaysia }\end{array}$ \\
\hline Ahmad, Mohd & University of Malaysia, Pahang \\
\hline \multicolumn{2}{|r|}{ Mexico } \\
\hline $\begin{array}{l}\text { Cedillo-Campos, } \\
\text { Miguel Gaston }\end{array}$ & Mexican Institute of Transport \\
\hline Cedillo, Gaston & $\begin{array}{l}\text { Mexican Logistics and Supply Chain } \\
\text { Association }\end{array}$ \\
\hline Lárraga, María & $\begin{array}{l}\text { National Autonomous University of } \\
\text { Mexico }\end{array}$ \\
\hline \multicolumn{2}{|r|}{ Montenegro } \\
\hline Bauk, Sanja & \multirow[t]{2}{*}{ University of Montenegro } \\
\hline Dragović, Branislav & \\
\hline \multicolumn{2}{|r|}{ Netherlands } \\
\hline Li, Zili & Delft University of Technology \\
\hline Veldman, Simme & ECORYS Netherlands \\
\hline Liao, Feixiong & Eindhoven University of Technology \\
\hline \multicolumn{2}{|r|}{ Norway } \\
\hline Klein-Paste, Alex & $\begin{array}{l}\text { Norwegian University of Science and } \\
\text { Technology }\end{array}$ \\
\hline Starykov, Maksym & Palfinger Marine Norway AS \\
\hline \multicolumn{2}{|r|}{ Pakistan } \\
\hline Abbas, Mazhar & University of Gujrat \\
\hline \multicolumn{2}{|r|}{ Panama } \\
\hline $\begin{array}{l}\text { Berbey Alvarez, } \\
\text { Aranzazu }\end{array}$ & \multirow[t]{2}{*}{ Technological University of Panama } \\
\hline $\begin{array}{l}\text { Quijada Alarcón, } \\
\text { Jorge }\end{array}$ & \\
\hline \multicolumn{2}{|r|}{ Poland } \\
\hline Gosiewski, Zdzisław & \multirow[t]{2}{*}{ Bialystok University of Technology } \\
\hline Szpica, Dariusz & \\
\hline $\begin{array}{l}\text { Bartłomiejczyk, } \\
\text { Mikołaj }\end{array}$ & Gdansk University of Technology \\
\hline Kossecki, Pawel & $\begin{array}{l}\text { Leon Schiller National Higher School } \\
\text { of Film, Television and Theatre }\end{array}$ \\
\hline Borkowski, Piotr & Lodz University of Technology \\
\hline Koszałka, Grzegorz & Lublin University of Technology \\
\hline Nowacki, Gabriel & \multirow[t]{2}{*}{ Military University of Technology } \\
\hline Prochowski, Leon & \\
\hline Paslawski, Jerzy & \multirow[t]{2}{*}{ Poznan University of Technology } \\
\hline Sawicki, Piotr & \\
\hline Krawiec, Stanisław & \multirow[t]{3}{*}{ Silesian University of Technology } \\
\hline Sitarz, Marek & \\
\hline $\begin{array}{l}\text { Sładkowski, } \\
\text { Aleksander }\end{array}$ & \\
\hline
\end{tabular}

\begin{tabular}{|c|c|}
\hline Surname, Name & Institution \\
\hline Tracz, Marian & $\begin{array}{l}\text { Tadeusz Kościuszko Cracow } \\
\text { University of Technology }\end{array}$ \\
\hline Bąk, Monika & University of Gdansk \\
\hline $\begin{array}{l}\text { Wierzbicki, } \\
\text { Slawomir }\end{array}$ & $\begin{array}{l}\text { University of Warmia and Mazury } \\
\text { in Olsztyn }\end{array}$ \\
\hline Witkowski, Jaroslaw & Wrocław University of Economics \\
\hline & Portugal \\
\hline $\begin{array}{l}\text { Marques, Rui } \\
\text { Cunha }\end{array}$ & Technical University of Lisbon \\
\hline Tchepel, Oxana & University of Aveiro \\
\hline Bastos Silva, Ana & \multirow[t]{3}{*}{ University of Coimbra } \\
\hline Bezerra, George & \\
\hline Ferreira, Adelino & \\
\hline $\begin{array}{l}\text { Alho, Andre } \\
\text { Romano }\end{array}$ & University of Lisbon \\
\hline $\begin{array}{l}\text { Costa Oliveira, José } \\
\text { Fernando }\end{array}$ & \multirow[t]{3}{*}{ University of Porto } \\
\hline Lobo, Antonio & \\
\hline Rosa-Santos, Paulo & \\
\hline \multicolumn{2}{|r|}{ Romania } \\
\hline $\begin{array}{l}\text { Bodea, Constanta- } \\
\text { Nicoleta }\end{array}$ & $\begin{array}{l}\text { Bucharest University of Economic } \\
\text { Studies }\end{array}$ \\
\hline Burnete, Nicolae & \multirow[t]{2}{*}{ Technical University of Cluj-Napoca } \\
\hline Mariasiu, Florin & \\
\hline \multicolumn{2}{|r|}{ Russia } \\
\hline Kulmanakov, Sergey & Altai State Technical University \\
\hline Panin, Sergey & $\begin{array}{l}\text { Institute of Strength Physics and } \\
\text { Materials Science of the Siberian } \\
\text { Branch of Russian Academy of } \\
\text { Sciences }\end{array}$ \\
\hline Andreev, Vladimir & $\begin{array}{l}\text { Moscow State University of Civil } \\
\text { Engineering }\end{array}$ \\
\hline Dyakov, Ivan & Ulyanovsk State Technical University \\
\hline \multicolumn{2}{|r|}{ Serbia } \\
\hline Stanujkić, Dragiša & Megatrend University \\
\hline Bojović, Nebojša & \multirow[t]{7}{*}{ University of Belgrade } \\
\hline Čokorilo, Olja & \\
\hline Jović, Jadranka & \\
\hline Milenković, Miloš & \\
\hline Pešić, Dalibor & \\
\hline Radmilović, Zoran & \\
\hline Vujanović, Davor & \\
\hline Petrović, Dragan & University in Kragujevac \\
\hline \multicolumn{2}{|r|}{ Singapore } \\
\hline Paw, Yew Chai & DSO National Laboratories \\
\hline Wang, David & Nanyang Technological University \\
\hline
\end{tabular}




\begin{tabular}{|c|c|}
\hline Surname, Name & Institution \\
\hline Choor, Chin Hoong & \multirow[t]{4}{*}{ National University of Singapore } \\
\hline Fang, Fwa Tien & \\
\hline Peng, Kemao & \\
\hline Xu, Jianxin & \\
\hline \multicolumn{2}{|r|}{ Slovakia } \\
\hline Adamčik, František & \multirow[t]{2}{*}{ Technical University of Košice } \\
\hline Guzanová, Anna & \\
\hline Barta, Dalibor & \multirow[t]{5}{*}{ University of Zilina } \\
\hline Bugaj, Martin & \\
\hline Gutten, Miroslav & \\
\hline Kasanický, Gustáv & \\
\hline Koháni, Michal & \\
\hline \multicolumn{2}{|r|}{ Slovenia } \\
\hline Bajec, Patricija & \multirow[t]{3}{*}{ University of Ljubljana } \\
\hline Batista, Milan & \\
\hline Twrdy, Elen & \\
\hline Klanšek, Uroš & \multirow[t]{4}{*}{ University of Maribor } \\
\hline Lerher, Tone & \\
\hline Tibaut, Andrej & \\
\hline Tollazzi, Tomaž & \\
\hline \multicolumn{2}{|r|}{ Spain } \\
\hline Godoy, Jorge & $\begin{array}{l}\text { Centre for Automation and Robotics, } \\
\text { AUTOPIA }\end{array}$ \\
\hline Onieva, Enrique & Deusto Institute of Technology \\
\hline Cabrera, Suitberto & Polytechnic University of Valencia \\
\hline Ayuga, Francisco & \multirow[t]{2}{*}{ Technical University of Madrid } \\
\hline Castro, Maria & \\
\hline Rojo, Marta & University of Burgos \\
\hline Alonso, Borja & \multirow[t]{2}{*}{ University of Cantabria } \\
\hline Dell'Olio, Luigi & \\
\hline Garach, Laura & University of Granada \\
\hline Muñuzuri, Jesús & University of Seville \\
\hline $\begin{array}{l}\text { Lenormand, } \\
\text { Maxime }\end{array}$ & University of the Balearic Islands \\
\hline $\begin{array}{l}\text { Acerete Gil, } \\
\text { José Basilio }\end{array}$ & University of Zaragoza \\
\hline \multicolumn{2}{|r|}{ Sweden } \\
\hline Linder, Astrid & $\begin{array}{l}\text { Swedish National Road and Transport } \\
\text { Research Institute }\end{array}$ \\
\hline Olsson, Jerry & University of Gothenburg \\
\hline \multicolumn{2}{|r|}{ Switzerland } \\
\hline Chen, Jiang Hang & $\begin{array}{l}\text { Swiss Federal Institute of Technology } \\
\text { in Lausanne }\end{array}$ \\
\hline Ciari, Francesco & \multirow{2}{*}{$\begin{array}{l}\text { Swiss Federal Institute of Technology } \\
\text { in Zurich }\end{array}$} \\
\hline Onder, Christopher & \\
\hline
\end{tabular}

\begin{tabular}{|c|c|}
\hline Surname, Name & Institution \\
\hline \multicolumn{2}{|r|}{ Taiwan } \\
\hline Hsu, Hui-Mi & National Ilan University \\
\hline Lin, Cherng-Yuan & \multirow[t]{2}{*}{ National Taiwan Ocean University } \\
\hline Shiau, Tzay-An & \\
\hline Yu, Vincent & $\begin{array}{l}\text { National Taiwan University of Science } \\
\text { and Technology }\end{array}$ \\
\hline \multicolumn{2}{|r|}{ Turkey } \\
\hline Çelik, Ali Kemal & Atatürk University \\
\hline $\begin{array}{l}\text { Rouyendegh, Babak } \\
\text { Daneshvar }\end{array}$ & Atılım University \\
\hline $\begin{array}{l}\text { Çalışkanelli, } \\
\text { Süheyla Pelin }\end{array}$ & \multirow[t]{2}{*}{ Dokuz Eylül University } \\
\hline Tanyel, Serhan & \\
\hline Demirel, Hande & \multirow[t]{3}{*}{ İstanbul Technical University } \\
\hline Kaya, Tolga & \\
\hline Turk, Sevkiye Sence & \\
\hline $\begin{array}{l}\text { Bezgin, Niyazi } \\
\text { Özgür }\end{array}$ & \multirow[t]{2}{*}{ İstanbul University } \\
\hline Karaşahin, Mustafa & \\
\hline Akgüngör, Ali & Kırıkkale University \\
\hline Ceylan, Halim & \multirow[t]{2}{*}{ Pamukkale University } \\
\hline Ceylan, Huseyin & \\
\hline Güler, Hakan & Sakarya University \\
\hline \multicolumn{2}{|r|}{ Ukraine } \\
\hline Perig, Alexander & Donbass State Engineering Academy \\
\hline $\begin{array}{l}\text { Bieliatynskyi, } \\
\text { Andrii }\end{array}$ & \multirow[t]{3}{*}{ National Aviation University } \\
\hline Glazunov, Nikolaj & \\
\hline $\begin{array}{l}\text { Krayushkina, } \\
\text { Kateryna }\end{array}$ & \\
\hline Klymenko, Iryna & National University of Transport \\
\hline Maruschak, Pavlo & $\begin{array}{l}\text { Ternopil Ivan Pul'uj National } \\
\text { Technical University }\end{array}$ \\
\hline \multicolumn{2}{|r|}{ United Arab Emirates } \\
\hline Ahmed, Kamran & United Arab Emirates University \\
\hline \multicolumn{2}{|c|}{ United Kingdom of Great Britain and Northern Ireland } \\
\hline Mason, Robert & Cardiff University \\
\hline Kanarachos, Stratis & Coventry University \\
\hline Velenis, Efstathios & Cranfield University \\
\hline Saleh, Wafaa & \multirow[t]{2}{*}{ Edinburgh Napier University } \\
\hline Stewart, Kathryn & \\
\hline Connolly, David & Heriot-Watt University \\
\hline Han, Ke & \multirow[t]{2}{*}{ Imperial College London } \\
\hline Krishnan, Rajesh & \\
\hline Eglese, Richard & $\begin{array}{l}\text { Lancaster University Management } \\
\text { School }\end{array}$ \\
\hline
\end{tabular}




\begin{tabular}{|c|c|}
\hline Surname, Name & Institution \\
\hline Li, Baibing & \multirow[t]{2}{*}{ Loughborough University } \\
\hline Ward, Christopher & \\
\hline Aditjandra, Paulus & \multirow[t]{3}{*}{ Newcastle University } \\
\hline Dunn, Sarah & \\
\hline Galatioto, Fabio & \\
\hline Briggs, Gemma & Open University \\
\hline Naeem, Wasif & Queen's University Belfast \\
\hline Cottrill, Caitlin & University of Aberdeen \\
\hline Erdoğan, Güneș & University of Bath \\
\hline $\begin{array}{l}\text { Kaewunruen, } \\
\text { Sakdirat }\end{array}$ & \multirow[t]{2}{*}{ University of Birmingham } \\
\hline $\begin{array}{l}\text { Wyszynski, } \\
\text { Miroslaw }\end{array}$ & \\
\hline Kashani, Mehdi & \multirow[t]{3}{*}{ University of Bristol } \\
\hline Shterenlikht, Anton & \\
\hline $\begin{array}{l}\text { Wanous, } \\
\text { Mohammed }\end{array}$ & \\
\hline Kinuthia, John & University of Glamorgan \\
\hline Iwnicki, Simon & University of Huddersfield \\
\hline \multicolumn{2}{|r|}{ United States } \\
\hline Baratian, Fatemeh & \multirow[t]{2}{*}{ Auburn University } \\
\hline $\begin{array}{l}\text { Pour-Rouholamin, } \\
\text { Mahdi }\end{array}$ & \\
\hline Wei, Junqing & Carnegie Mellon University \\
\hline Xiong, Qingang & Iowa State University \\
\hline Cornillie, Thomas & Independent Scholar \\
\hline Ji, Yigong & $\begin{array}{l}\text { Indiana Department } \\
\text { of Transportation }\end{array}$ \\
\hline $\begin{array}{l}\text { Gopalakrishnan, } \\
\text { Kasthurirangan }\end{array}$ & \multirow[t]{3}{*}{ Iowa State University } \\
\hline Sharma, Anuj & \\
\hline Smadi, Omar & \\
\hline $\begin{array}{l}\text { Santhanam, Ganesh } \\
\text { Ram }\end{array}$ & $\begin{array}{l}\text { Iowa State University of Science } \\
\text { and Technology }\end{array}$ \\
\hline $\begin{array}{l}\text { Daphalapurkar, } \\
\text { Nitin }\end{array}$ & Johns Hopkins University \\
\hline Xiao, Danny & Louisiana State University \\
\hline Zoepf, Stephen & Massachusetts Institute of Technology \\
\hline Vatavu, Andrei & Mercedes-Benz R\&D North America \\
\hline Lautala, Pasi & \multirow[t]{2}{*}{ Michigan Technological University } \\
\hline Pouryousef, Hamed & \\
\hline Newman, James & Mississippi State University \\
\hline Yager, Thomas & $\begin{array}{l}\text { National Aeronautics and Space } \\
\text { Administration (NASA) }\end{array}$ \\
\hline Chien, Steven & New Jersey Institute of Technology \\
\hline Agrawal, Ankit & Northwestern University \\
\hline
\end{tabular}

\begin{tabular}{|c|c|}
\hline Surname, Name & Institution \\
\hline Ye, Fan & Ohio Northern University \\
\hline Donnell, Eric T. & Penn State University \\
\hline Brownell, Chris & Princeton University \\
\hline Bullough, John & Rensselaer Polytechnic Institute \\
\hline $\begin{array}{l}\text { Yadollahi, } \\
\text { Mohammadreza }\end{array}$ & Rowan University \\
\hline Deka, Devajyoti & \multirow[t]{2}{*}{ Rutgers University } \\
\hline Yi, Jingang & \\
\hline Liu, Litao & Texas A\&M Transportation Institute \\
\hline Duru, Okan & Texas A\&M University at Galveston \\
\hline $\begin{array}{l}\text { Nachtmann, } \\
\text { Heather Lyn }\end{array}$ & University of Arkansas \\
\hline Yang, Xianfeng & University of Maryland \\
\hline Shaheen, Susan & \multirow[t]{2}{*}{ University of California, Berkeley } \\
\hline Wei, Dali & \\
\hline Lea, Jeremy D. & \multirow[t]{2}{*}{ University of California, Davis } \\
\hline Oum, Sanghyouk & \\
\hline Garrick, Norman & University of Connecticut \\
\hline James, Leon & University of Hawai'i System \\
\hline Zhao, Ming & University of Houston \\
\hline Fitzsimmons, Eric & University of Kansas \\
\hline Gårder, Per & University of Maine \\
\hline Golias, Mihalis & University of Memphis \\
\hline Mudgal, Abhisek & University of Minnesota \\
\hline Hajj, Elie & University of Nevada, Reno \\
\hline Qadir, Ashraf & University of North Dakota \\
\hline Dong, Chunjiao & University of Tennessee \\
\hline Di, Long & University of Virginia \\
\hline Li, Zhixia & University of Wisconsin-Madison \\
\hline & Vietnam \\
\hline Viet, La Duc & $\begin{array}{l}\text { Institute of Mechanics, Vietnam } \\
\text { Academy of Science and Technology }\end{array}$ \\
\hline
\end{tabular}

